\title{
Oscillation and nonoscillation for second order neutral dynamic equations with positive and negative coefficients on time scales
}

Xun-Huan Deng ${ }^{1}$, Qi-Ru Wang ${ }^{1}$ and Ravi P Agarwal $2,3^{*}$

${ }^{*}$ Correspondence:

Agarwal@tamuk.edu

2Department of Mathematics, Texas

A\&M University-Kingsville,

700 University Blvd., Kingsville,

TX 78363-8202, USA

${ }^{3}$ Department of Mathematics, King

Abdulaziz University, P.O. Box 80203,

Jeddah, 21589, Saudi Arabia

Full list of author information is

available at the end of the article

\begin{abstract}
We investigate oscillation and nonoscillation of certain second order neutral dynamic equations with positive and negative coefficients. We apply the results from the theory of lower and upper solutions for related dynamic equations along with some additional estimates on positive solutions and use different techniques to obtain some oscillatory theorems. Also, we apply Kranoselskii's fixed point theorem to obtain nonoscillatory results and then give two sufficient and necessary conditions for the equations to be oscillatory. Some interesting examples are given to illustrate the versatility of our results.
\end{abstract}

Keywords: neutral dynamic equations; positive and negative coefficients; oscillation and nonoscillation; lower and upper solutions; Kranoselskii's fixed point theorem

\section{Introduction}

In this paper, we investigate oscillation and nonoscillation of second order neutral functional dynamic equations with positive and negative coefficients of the form

$$
\left[p(t)(x(t)+r(t) x(g(t)))^{\Delta}\right]^{\Delta}+h\left(t, x^{\sigma}(t), x\left(\tau_{1}(t)\right), x\left(\tau_{2}(t)\right), x\left(\xi_{1}(t)\right), x\left(\xi_{2}(t)\right)\right)=0,
$$

where $t \in \mathbb{T}, h \in C\left(\mathbb{T} \times \mathbb{R}^{1+2+2}, \mathbb{R}\right), \Phi_{\gamma}(u)=|u|^{\gamma-1} u, \gamma>0$,

$$
\begin{aligned}
h\left(t, x_{0}, x_{1}, x_{2}, x_{3}, x_{4}\right)= & q_{0}(t) \Phi_{\gamma}\left(x_{0}\right)+q_{1}(t) \Phi_{\gamma}\left(x_{1}\right)-q_{2}(t) \Phi_{\gamma}\left(x_{2}\right) \\
& +s_{1}(t) \Phi_{\gamma}\left(x_{3}\right)-s_{2}(t) \Phi_{\gamma}\left(x_{4}\right) .
\end{aligned}
$$

Throughout this paper, we shall assume that $\mathbb{T}$ is a time scale satisfying inf $\mathbb{T}=t_{0}$ and $\sup \mathbb{T}=\infty$, and

(B1) $p \in C_{\mathrm{rd}}\left(\left[t_{0}, \infty\right)_{\mathbb{T}},(0, \infty)\right)$ satisfies $\int_{t_{0}}^{\infty} \frac{1}{p(s)} \Delta s=\infty$;

(B2) there exists a constant $r_{0}, 0 \leq r_{0}<1$ such that $r \in C_{\mathrm{rd}}\left(\mathbb{T},\left[0, r_{0}\right]\right)$;

(B3) $g \in C_{\mathrm{rd}}(\mathbb{T}, \mathbb{T}), g(t) \leq t, \lim _{t \rightarrow \infty} g(t)=\infty$;

(B4) $\tau_{1}, \tau_{2} \in C_{\mathrm{rd}}(\mathbb{T}, \mathbb{T})$ are injective, $\tau_{1}(t) \leq \tau_{2}(t) \leq t, \lim _{t \rightarrow \infty} \tau_{1}(t)=\infty$ and $\operatorname{Im} \tau_{1}(\mathbb{T}) \supseteq \operatorname{Im} \tau_{2}(\mathbb{T})$ 
(B5) $\xi_{1}, \xi_{2} \in C_{\mathrm{rd}}(\mathbb{T}, \mathbb{T})$ are injective, $\xi_{1}(t) \geq \xi_{2}(t) \geq \sigma(t)$, and for sufficiently large $T_{0} \in \mathbb{T}$, there exists $T_{0}^{\prime} \in \mathbb{T}$ such that $\xi_{1}\left(T_{0}\right)=\xi_{2}\left(T_{0}^{\prime}\right)$ and $A_{1}=\left\{\operatorname{Im} \xi_{1}(t): t \geq T_{0}\right\} \supseteq A_{2}=\left\{\operatorname{Im} \xi_{2}(t): t \geq T_{0}^{\prime}\right\}$

(B6) $q_{0}(t), q_{1}(t), q_{2}(t), s_{1}(t), s_{2}(t) \in C_{\mathrm{rd}}(\mathbb{T}, \mathbb{R})$ are eventually positive, and satisfy

$$
\begin{array}{ll}
q_{1}\left(\tau_{1}^{-1}\left(\tau_{2}(t)\right)\right) \geq q_{2}(t), & s_{1}\left(\xi_{1}^{-1}\left(\xi_{2}(t)\right)\right) \geq s_{2}(t), \\
q_{0}\left(\sigma^{-1}\left(\xi_{2}(t)\right)\right) \geq s_{2}(t), & q_{0}\left(\sigma^{-1}\left(\tau_{1}(t)\right)\right) \geq q_{2}(t) .
\end{array}
$$

A solution $x(t)$ of (1.1) is said to be oscillatory if it is neither eventually positive nor eventually negative, otherwise it is nonoscillatory. The equation itself is called oscillatory if all its solutions are oscillatory.

In recent years, there has been an increasing interest in studying the oscillation and nonoscillation of solutions of dynamic equations on time scales since Hilger introduced the theory of time scale which was excepted to unify continuous and discrete calculus. We refer the readers to the monographs [1-4], the papers [3,5-17] and the references cited therein.

The results on oscillation of dynamic equations with positive and negative coefficients are mainly concentrated on differential equations or difference equations. To the best of our knowledge, there are few researches on dynamic equations with positive and negative coefficients on time scales. In [13], Özbekler and Zafer gave new oscillation criteria for superlinear and sublinear forced dynamic equations with positive and negative coefficients by means of nonprinciple solutions. Also, Özbekler et al. [14] made use of the concept of nonprinciple solutions to establish new oscillation criteria. However, in general, it is difficult for us to find a nonprinciple solution of second order dynamic equations. As a result, their approach may be difficult to apply to second order dynamic equations. Under the convergence of double integral of negative coefficients, sufficient conditions for oscillation was given in $[12,16-18]$. The results on oscillation of difference equations with positive and negative coefficients can be found in $[8,15]$ and references therein.

In this paper, to obtain oscillatory theorems, we shall apply results from the theory of lower and upper solutions for related dynamic equations along with some additional estimates on positive solutions and use some different techniques. Also, we apply Kranoselskii's fixed point theorem to obtain nonoscillatory results and then give two sufficient and necessary conditions for (1.1) being oscillatory. Our results cannot only be applied to differential equations and difference equations, but they can also be applied to other dynamic equations with positive and negative coefficients.

In Section 2, we present some preliminaries and important estimates, especially the estimate $z^{\Delta}(t) \geq 0$ and the function $h>0$ if the solution $x(t)>0$ of (1.1). In Section 3, we give several oscillatory and nonoscillatory results. In Section 4, we illustrate the versatility of our results by three examples.

\section{Some preliminaries}

In order to prove our main results, we establish some fundamental results in this section. Now we introduce the auxiliary functions

$$
P(t, a)=\int_{a}^{t} \frac{\Delta s}{p(s)}, \quad \eta_{i}(t, a)=\frac{P\left(\tau_{i}(t), a\right)}{P(\sigma(t), a)}, \quad v_{j}(t, a)=\frac{P\left(\xi_{j}(t), a\right)}{P(\sigma(t), a)},
$$


where $1 \leq i \leq n, 1 \leq j \leq m, a \in\left[t_{0}, \infty\right)_{\mathbb{T}}$. For the convenience of discussion, let

$$
z(t)=x(t)+r(t) x(g(t)) .
$$

Let $m_{1}=1-r_{0}$. Furthermore, we need the following additional hypotheses:

$$
q_{0}(t) \Phi_{\gamma}\left(m_{1}\right)-q_{2}(t) \geq 0, \quad s_{1}(t) \Phi_{\gamma}\left(m_{1}\right)-s_{2}(t) \geq 0
$$

or

$$
s_{1}(t) \Phi_{\gamma}\left(m_{1}\right)-q_{2}(t)-s_{2}(t) \geq 0 .
$$

First of all, we give the following estimates.

Lemma 2.1 Assume that conditions (B1)-(B6) and one of (2.2)-(2.3) hold. Let $x(t)$ be an eventually positive solution of equation (1.1). Then there exists some $T \geq t_{0}$ such that

(i) for all $t \geq T, z(t)>0, z^{\Delta}(t)>0, x(t) \geq(1-r(t)) z(t)$, and

$$
h\left(t, x^{\sigma}(t), x\left(\tau_{1}(t)\right), x\left(\tau_{2}(t)\right), x\left(\xi_{1}(t)\right), x\left(\xi_{2}(t)\right)\right)>0 ;
$$

(ii) for each $1 \leq i \leq 2,1 \leq j \leq 2$ and for $\xi_{j}(t) \geq \sigma(t) \geq t \geq \tau_{i}(t) \geq T$, we have

$$
z\left(\tau_{i}(t)\right) \geq \eta_{i}(t, T) z^{\sigma}(t) \quad \text { and } \quad z\left(\xi_{j}(t)\right) \leq v_{j}(t, T) z^{\sigma}(t)
$$

(iii) if $p(t)$ is nondecreasing, each $1 \leq i \leq 2,1 \leq j \leq 2$ and for $\xi_{j}(t) \geq \sigma(t) \geq t \geq \xi_{j}(T) \geq \tau_{i}(t) \geq T$, we have

$$
\frac{z\left(\tau_{i}(t)\right)}{z^{\sigma}(t)} \geq \frac{\tau_{i}(t)-\tau_{i}(T)}{\sigma(t)-\tau_{i}(T)} \quad \text { and } \quad \frac{z\left(\xi_{j}(t)\right)}{z^{\sigma}(t)} \leq \frac{\xi_{j}(t)-\xi_{j}(T)}{\sigma(t)-\xi_{j}(T)} .
$$

Proof (i) Suppose that $x(t)$ is an eventually positive solution of (1.1). In view of conditions (B2)-(B6), there exists $T_{1} \in \mathbb{T}$ such that $x(t)>0, x(g(t))>0, x\left(\tau_{i}(t)\right)>0,1 \leq i \leq 2, x\left(\xi_{j}(t)\right)>$ $0,1 \leq j \leq 2$, and $0 \leq r(t) \leq r_{0}<1$ for all $t \in\left[T_{1}, \infty\right)_{\mathbb{T}}$. It is immediate to obtain $z(t) \geq 0$ by $z(t)=x(t)+r(t) x(g(t))$.

Next, we show that $z^{\Delta}(t)$ is eventually positive. Otherwise there exists a sequence $\left\{t_{n}\right\}_{n=1}^{\infty}$ with $\lim _{l \rightarrow \infty} t_{l}=\infty$ such that $z^{\Delta}\left(t_{i}\right)<0$ and $\tau_{1}^{-1}\left(\tau_{2}\left(t_{l}\right)\right) \geq t_{l}$ for some $l \in \mathbb{N}$. Without loss of generality, we may assume that $l=1$, i.e. $\tau_{1}^{-1}\left(\tau_{2}\left(t_{1}\right)\right) \geq t_{1}$. For $t \geq \tau_{2}^{-1}\left(\tau_{1}(t)\right) \geq \xi_{2}^{-1}\left(\xi_{1}\left(t_{1}\right)\right) \geq t_{1}$ and $t \geq \xi_{1}^{-1}\left(\xi_{2}(t)\right)$, let

$$
\left\{\begin{aligned}
D_{1}= & -\int_{t_{1}}^{t} q_{0}(s) \Phi_{\gamma}\left(x^{\sigma}(s)\right) \Delta s+\int_{\tau_{2}^{-1}\left(\tau_{1}(t)\right)}^{t} q_{2}(s) \Phi_{\gamma}\left(x\left(\tau_{2}(s)\right)\right) \Delta s \\
& +\int_{t_{1}}^{\xi_{2}^{-1}\left(\xi_{1}\left(t_{1}\right)\right)} s_{2}(s) \Phi_{\gamma}\left(x\left(\xi_{2}(s)\right)\right) \Delta s, \\
D_{2}= & -\int_{t_{1}}^{t} q_{1}(s) \Phi_{\gamma}\left(x\left(\tau_{1}(s)\right)\right) \Delta s+\int_{t_{1}}^{\tau_{2}^{-1}\left(\tau_{1}(t)\right)} q_{2}(s) \Phi_{\gamma}\left(x\left(\tau_{2}(s)\right)\right) \Delta s, \\
D_{3}= & -\int_{t_{1}}^{t} s_{1}(s) \Phi_{\gamma}\left(x\left(\xi_{1}(s)\right)\right) \Delta s+\int_{\xi_{2}^{-1}\left(\xi_{1}\left(t_{1}\right)\right)}^{t} s_{2}(s) \Phi_{\gamma}\left(x\left(\xi_{2}(s)\right)\right) \Delta s .
\end{aligned}\right.
$$

We rewrite $D_{2}$ as follows:

$$
\begin{aligned}
D_{2}= & -\int_{t_{1}}^{\tau_{1}^{-1}\left(\tau_{2}\left(t_{1}\right)\right)} q_{1}(s) \Phi_{\gamma}\left(x\left(\tau_{1}(s)\right)\right) \Delta s-\int_{\tau_{1}^{-1}\left(\tau_{2}\left(t_{1}\right)\right)}^{t} q_{1}(s) \Phi_{\gamma}\left(x\left(\tau_{1}(s)\right)\right) \Delta s \\
& +\int_{t_{1}}^{\tau_{2}^{-1}\left(\tau_{1}(t)\right)} q_{2}(s) \Phi_{\gamma}\left(x\left(\tau_{2}(s)\right)\right) \Delta s .
\end{aligned}
$$


By (B6), we see that $q_{1}\left(\tau_{1}^{-1}\left(\tau_{2}(t)\right)\right) \geq q_{2}(t)$ and then clearly $D_{2} \leq 0$. Similarly, we also have $D_{1} \leq 0$ and $D_{3} \leq 0$.

Then, for $t \geq \tau_{2}^{-1}\left(\tau_{1}(t)\right) \geq \xi_{2}^{-1}\left(\xi_{1}\left(t_{1}\right)\right) \geq t_{1}$ and $t \geq \xi_{1}^{-1}\left(\xi_{2}(t)\right)$, integrating (1.1) from $t_{1}$ to $t$, by (B1)-(B6) and the geometric sense of a definite integral, we obtain

$$
\begin{aligned}
p(t) z^{\Delta}(t)-p\left(t_{1}\right) z^{\Delta}\left(t_{1}\right) & =-\int_{t_{1}}^{t} h\left(s, x^{\sigma}(s), x\left(\tau_{1}(s)\right), x\left(\tau_{2}(s)\right), x\left(\xi_{1}(s)\right), x\left(\xi_{2}(s)\right)\right) \Delta s \\
& =D_{1}+D_{2}+D_{3} \leq 0 .
\end{aligned}
$$

It follows that

$$
z(t)-z\left(t_{1}\right) \leq p\left(t_{1}\right) z^{\Delta}\left(t_{1}\right) \int_{t_{1}}^{t} \frac{\Delta s}{p(s)} \rightarrow-\infty \quad \text { as } t \rightarrow \infty,
$$

which contradicts $z(t)=x(t)+r(t) x(g(t))>0$.

Since $x(t)$ is a solution of (1.1) satisfying $x(t)>0$ and $z^{\Delta}(t)>0$, we see that

$$
x(t)=z(t)-r(t) x(g(t)) \geq z(t)-r(t) z(g(t)) \geq z(t)-r(t) z(t)=(1-r(t)) z(t) .
$$

Now, we claim that $h\left(t, x^{\sigma}(t), x\left(\tau_{1}(t)\right), x\left(\tau_{2}(t)\right), x\left(\xi_{1}(t)\right), x\left(\xi_{2}(t)\right)\right)>0$. By (2.2) or (2.3), $x(t) \geq(1-r(t)) z(t)$ and $z^{\Delta}(t) \geq 0$ for $t \geq T_{1}$, we have

$$
\begin{aligned}
h\left(t, x^{\sigma}(t), x\left(\tau_{1}(t)\right), x\left(\tau_{2}(t)\right), x\left(\xi_{1}(t)\right), x\left(\xi_{2}(t)\right)\right) \\
=q_{0}(t) \Phi_{\gamma}\left(x^{\sigma}(t)\right)+\sum_{i=1}^{2}(-1)^{i-1} q_{i}(t) \Phi_{\gamma}\left(x\left(\tau_{i}(t)\right)\right)+\sum_{i=1}^{2}(-1)^{i-1} s_{i}(t) \Phi_{\gamma}\left(x\left(\xi_{i}(t)\right)\right) \\
\geq q_{0}(t) \Phi_{\gamma}\left(m_{1} z^{\sigma}(t)\right)+q_{1}(t) \Phi_{\gamma}\left(m_{1} z\left(\tau_{1}(t)\right)\right)-q_{2}(t) \Phi_{\gamma}\left(z\left(\tau_{2}(t)\right)\right) \\
\quad+s_{1}(t) \Phi_{\gamma}\left(m_{1} z\left(\xi_{1}(t)\right)\right)-s_{2}(t) \Phi_{\gamma}\left(z\left(\xi_{2}(t)\right)\right)>0 .
\end{aligned}
$$

(ii) It is clear that $\left[p(t) z^{\Delta}(t)\right]^{\Delta}<0$ or $p(t) z^{\Delta}(t)$ is nonincreasing since $h>0$ for $t \geq T$. For $t \geq \tau_{i}(t) \geq T \geq t_{0}$ and $1 \leq i \leq 2$, we have

$$
z^{\sigma}(t)-z\left(\tau_{i}(t)\right)=\int_{\tau_{i}(t)}^{\sigma(t)} \frac{1}{p(s)} p(s) z^{\Delta}(s) \Delta s \leq p\left(\tau_{i}(t)\right) z^{\Delta}\left(\tau_{i}(t)\right) \int_{\tau_{i}(t)}^{\sigma(t)} \frac{1}{p(s)} \Delta s .
$$

Dividing both sides of above inequality by $z\left(\tau_{i}(t)\right)$, we obtain

$$
\frac{z^{\sigma}(t)}{z\left(\tau_{i}(t)\right)} \leq 1+\frac{p\left(\tau_{i}(t)\right) z^{\Delta}\left(\tau_{i}\right)}{z\left(\tau_{i}(t)\right)} P\left(\sigma(t), \tau_{i}(t)\right) .
$$

Likewise, we also have

$$
z\left(\tau_{i}(t)\right)-z(T)=\int_{T}^{\tau_{i}(t)} \frac{1}{p(s)} p(s) z^{\Delta}(s) \Delta s \geq p\left(\tau_{i}(t)\right) z^{\Delta}\left(\tau_{i}(t)\right) \int_{T}^{\tau_{i}(t)} \frac{1}{p(s)} \Delta s
$$

and

$$
\frac{p\left(\tau_{i}(t)\right) z^{\Delta}\left(\tau_{i}(t)\right)}{z\left(\tau_{i}(t)\right)} \leq \frac{1}{P\left(\tau_{i}(t), T\right)} .
$$


Hence, (2.4) and (2.5) imply

$$
\frac{z^{\sigma}(t)}{z\left(\tau_{i}(t)\right)} \leq 1+\frac{p\left(\tau_{i}(t)\right) z^{\Delta}\left(\tau_{i}\right)}{z\left(\tau_{i}(t)\right)} P\left(\sigma(t), \tau_{i}(t)\right) \leq \frac{P(\sigma(t), T)}{P\left(\tau_{i}(t), T\right)}
$$

This gives the desired result

$$
z\left(\tau_{i}(t)\right) \geq \eta_{i}(t, T) z^{\sigma}(t), \quad 1 \leq i \leq 2 .
$$

For $T<t \leq \sigma(t) \leq \xi_{j}(t), 1 \leq j \leq 2$, we also get

$$
\left\{\begin{array}{l}
\frac{z\left(\xi_{j}(t)\right)}{z^{\sigma}(t)} \leq 1+\frac{p(\sigma(t)) z^{\Delta}(\sigma(t))}{z^{\sigma}(t)} P\left(\xi_{j}(t), \sigma(t)\right), \\
\frac{z^{\sigma}(t)}{p(\sigma(t)) z^{\Delta}(\sigma(t))} \geq \frac{z(T)}{p(\sigma(t)) z^{\Delta}(\sigma(t))}+P(\sigma(t), T) .
\end{array}\right.
$$

Therefore, we obtain

$$
\frac{z\left(\xi_{j}(t)\right)}{z^{\sigma}(t)} \leq 1+\frac{p(\sigma(t)) z^{\Delta}(\sigma(t))}{z^{\sigma}(t)} P\left(\xi_{j}(t), \sigma(t)\right) \leq 1+\frac{P\left(\xi_{j}(t), \sigma(t)\right)}{P(\sigma(t), T)}=v_{j}(t, T),
$$

which yields the desired result

$$
z\left(\xi_{j}(t)\right) \leq z^{\sigma}(t) v_{j}(t, T)
$$

(iii) It can be proved similar to [7, Lemma 2.1] and hence its proof is omitted here.

In addition to the above lemmas, we need a method of studying separated boundary value problems (SBVP) to prove our main results. Namely, we will define functions called upper and lower solutions that not only imply the existence of a solution of a SBVP, but that also provide bounds on the location of the solution. Consider the SBVP

$$
\left\{\begin{array}{l}
-\left(p(t) y^{\Delta}(t)\right)^{\Delta}+q(t) y^{\sigma}=f\left(t, y^{\sigma}\right), \quad t \in[a, b]^{\kappa^{2}} \\
y(a)=A, \quad y(b)=B
\end{array}\right.
$$

where the functions $f \in C\left([a, b]^{\kappa^{2}} \times \mathbb{R}, \mathbb{R}\right), p, q \in C\left([a, b]^{\kappa^{2}}\right)$ with $p(t)>0$ and $q(t) \geq 0$ on $[a, b]^{\kappa^{2}}$. We define the set

$$
\mathbb{D}_{1}:=\left\{y \in \mathbb{X}: y^{\Delta} \text { is continuous and }\left(p y^{\Delta}\right)^{\Delta} \text { is rd-continuous on }[a, b]^{\kappa^{2}}\right\},
$$

where the Banach space $\mathbb{X}=C\left([a, b]_{\mathbb{T}}\right)$ is equipped with the norm $\|\bullet\|$ defined by

$$
\|y\|:=\max _{t \in[a, b]_{\mathbb{T}}}|y(t)| \quad \text { for all } y \in \mathbb{X} .
$$

A function $y$ is called a solution of the equation $-\left(p(t) y^{\Delta}(t)\right)^{\Delta}+q(t) y^{\sigma}=0$, on $[a, b]^{\kappa^{2}}$ if $y \in \mathbb{D}_{1}$ and the equation $-\left(p(t) y^{\Delta}(t)\right)^{\Delta}+q(t) y^{\sigma}=0$ holds for all $t \in[a, b]^{\kappa^{2}}$. Next, we define for any $u, v \in \mathbb{D}_{1}$ the sector $[u, v]_{1}$ by

$$
[u, v]_{1}:=\left\{w \in \mathbb{D}_{1} \mid u \leq w \leq v\right\} .
$$


Definition 2.1 [3, Definition 6.1] We call $\alpha \in \mathbb{D}_{1}$ a lower solution of the SBVP (2.6)-(2.7) on $[a, b]_{\mathbb{T}}$ provided

$$
\left\{\begin{array}{l}
-\left(p(t) \alpha^{\Delta}(t)\right)^{\Delta}+q(t) \alpha^{\sigma}(t) \leq f\left(t, \alpha^{\sigma}(t)\right) \quad \text { for all } t \in[a, b]^{\kappa^{2}} \\
\alpha(a) \leq A, \quad \alpha(b) \leq B
\end{array}\right.
$$

Similarly, $\beta \in \mathbb{D}_{1}$ is called an upper solution of the $\operatorname{SBVP}(2.6)-(2.7)$ on $[a, b]$ provided

$$
\left\{\begin{array}{l}
-\left(p(t) \beta^{\Delta}(t)\right)^{\Delta}+q(t) \beta^{\sigma}(t) \geq f\left(t, \beta^{\sigma}(t)\right) \quad \text { for all } t \in[a, b]^{\kappa^{2}}, \\
\beta(a) \geq A, \quad \beta(b) \geq B .
\end{array}\right.
$$

The following theorem is an extension of $[3$, Theorem 6.5$]$ to $[a, \infty)_{\mathbb{T}}$.

Theorem 2.1 [10, Theorem 1.5] Assume that there exist a lower solution $\alpha$ and an upper solution $\beta$ of $(2.6)$ with $\alpha(t) \leq \beta(t)$ for all $t \in[a, \infty)_{\mathbb{T}}$. Then

$$
-\left(p(t) y^{\Delta}(t)\right)^{\Delta}+q(t) y^{\sigma}=f\left(t, y^{\sigma}\right)
$$

has a solution $y$ with $y(a)=A$ and $y \in[\alpha, \beta]_{1}$ on $[a, \infty)_{\mathbb{T}}$.

We end this section with time scale version of the Arzelà-Ascoli theorem (see [7, Lemma 2.2]) and Kranoselskii's fixed point theorem (see [7]). These will be used in the proof of Theorem 3.3.

For $T_{0}, T_{1} \in \mathbb{T}$, let $\left[T_{0}, \infty\right)_{\mathbb{T}}:=\left\{t \in \mathbb{T}: t \geq T_{0}\right\}$ and $\left[T_{0}, T_{1}\right]_{\mathbb{T}}:=\left\{t \in \mathbb{T}: T_{0} \leq t \leq T_{1}\right\}$. Further, let $C\left(\left[T_{0}, \infty\right)_{\mathbb{T}}, \mathbb{R}\right)$ denote all continuous functions mapping $\left[T_{0}, \infty\right)_{\mathbb{T}}$ into $\mathbb{R}$,

$$
B C\left[T_{0}, \infty\right)_{\mathbb{T}}:=\left\{x: x \in C\left(\left[T_{0}, \infty\right)_{\mathbb{T}}, \mathbb{R}\right) \text { and } \sup _{t \in\left[T_{0}, \infty\right)_{\mathbb{T}}}|x(t)|<\infty\right\} .
$$

Endowed on $B C\left[T_{0}, \infty\right)_{\mathbb{T}}$ with the norm $\|x\|=\sup _{t \in\left[T_{0}, \infty\right)_{\mathbb{T}}}|x(t)|,\left(B C\left[T_{0}, \infty\right)_{\mathbb{T}},\|\bullet\|\right)$ is a Banach space. Let $X \subseteq B C\left[T_{0}, \infty\right)_{\mathbb{T}}$, we say $X$ is uniformly Cauchy if for any given $\varepsilon>0$, there exists a $T_{1} \in\left[T_{0}, \infty\right)_{\mathbb{T}}$ such that for any $x \in X$,

$$
\left|x\left(t_{1}\right)-x\left(t_{2}\right)\right|<\varepsilon \quad \text { for all } t_{1}, t_{2} \in\left[T_{1}, \infty\right)_{\mathbb{T}} .
$$

$X$ is said to be equi-continuous on $[a, b]_{\mathbb{T}}$ if for any given $\varepsilon>0$, there exists a $\delta>0$ such that for any $x \in X$ and $t_{1}, t_{2} \in[a, b]_{\mathbb{T}}$ with $\left|t_{1}-t_{2}\right|<\delta$,

$$
\left|x\left(t_{1}\right)-x\left(t_{2}\right)\right|<\varepsilon \text {. }
$$

Lemma 2.2 [7, Lemma 2.2] Suppose that $X \subseteq B C\left[T_{0}, \infty\right)_{\mathbb{T}}$ is bounded and uniformly Cauchy. Further, suppose that $X$ is equi-continuous on $\left[T_{0}, T_{1}\right]_{\mathbb{T}}$ for any $T_{1} \in\left[T_{0}, \infty\right)_{\mathbb{T}}$. Then $X$ is relatively compact.

Lemma 2.3 (Kranoselskii's fixed point theorem) Suppose that $X$ is a Banach space and $\Omega$ is a bounded, convex and closed subset of $X$. Suppose further that there exist two operators $U, S: \Omega \rightarrow X$ such that 
(i) $U x+$ Sy $\in \Omega$ for all $x, y \in \Omega$;

(ii) $U$ is a contraction mapping;

(iii) $S$ is completely continuous.

Then $U+S$ has a fixed point in $\Omega$.

\section{Main results}

In this section, we establish our main results.

Theorem 3.1 Assume conditions (B1)-(B6) and one of (2.2)-(2.3) hold. Then any bounded solution $x(t)$ of $(1.1)$ is oscillatory in the case

$$
\left|\int^{\infty} P(t, a) h\left(t, m_{1} \alpha, m_{1} \alpha \eta_{1}, \alpha, m_{1} \alpha, \alpha\right) \Delta t\right|=\infty
$$

for all $\alpha \neq 0$ and some sufficiently large $a \in \mathbb{T}$, where $m_{1}=1-r_{0}, \eta_{1}=\eta_{1}(t, a)$ is given in (2.1).

Proof Assume this not to be the case and let $u(t)$ be a bounded nonoscillatory solution of (1.1) which we may assume satisfies

$$
u(t)>0, \quad u\left(\tau_{i}(t)\right)>0, \quad u\left(\xi_{j}(t)\right)>0, \quad t \geq T \geq t_{0}, 1 \leq i \leq 2,1 \leq j \leq 2 .
$$

For convenience, let $z_{1}(t)=u(t)+r(t) u(g(t))$. By Lemma 2.1, we have

$$
z_{1}^{\Delta}(t) \geq 0, \quad u(t) \geq(1-r(t)) z_{1}(t), \quad z_{1}\left(\tau_{1}(t)\right) \geq \eta_{1}(t, T) z_{1}^{\sigma}(t) .
$$

Define the function $H\left(t, \theta_{1}\right)$ as follows:

$$
H\left(t, \theta_{1}\right):=h\left(t, m_{1} \theta_{1}, m_{1} \eta_{1} \theta_{1}, \theta_{1}, m_{1} \theta_{1}, \theta_{1}\right) .
$$

On one hand, by (2.2), Lemma 2.1, (B4)-(B6), the monotonicity of $\Phi_{\gamma}(u), z_{1}^{\Delta}(t) \geq 0$, and $\eta_{1}=\eta_{1}(t, T)$, for $t \geq T$, we have

$$
\begin{aligned}
0= & {\left[p(t) z_{1}^{\Delta}(t)\right]^{\Delta}+h\left(t, u^{\sigma}(t), u\left(\tau_{1}(t)\right), u\left(\tau_{2}(t)\right), u\left(\xi_{1}(t)\right), u\left(\xi_{2}(t)\right)\right) } \\
\geq & {\left[p(t) z_{1}^{\Delta}(t)\right]^{\Delta}+q_{0}(t) \Phi_{\gamma}\left(m_{1} z_{1}^{\sigma}(t)\right)+q_{1}(t) \Phi_{\gamma}\left(m_{1} \eta_{1} z_{1}^{\sigma}(t)\right)-q_{2}(t) \Phi_{\gamma}\left(z_{1}^{\sigma}(t)\right) } \\
& +s_{1}(t) \Phi_{\gamma}\left(m_{1} z_{1}\left(\xi_{1}(t)\right)\right)-s_{2}(t) \Phi_{\gamma}\left(z_{1}\left(\xi_{2}(t)\right)\right) \\
\geq & {\left[p(t) z_{1}^{\Delta}(t)\right]^{\Delta}+q_{0}(t) \Phi_{\gamma}\left(m_{1} z_{1}^{\sigma}(t)\right)+q_{1}(t) \Phi_{\gamma}\left(m_{1} \eta_{1} z_{1}^{\sigma}(t)\right)-q_{2}(t) \Phi_{\gamma}\left(z_{1}^{\sigma}(t)\right) } \\
& +\left[s_{1}(t) \Phi_{\gamma}\left(m_{1}\right)-s_{2}(t)\right] \Phi_{\gamma}\left(z_{1}\left(\xi_{1}(t)\right)\right) \\
= & {\left[p(t) z_{1}^{\Delta}(t)\right]^{\Delta}+\left[q_{0}(t) \Phi_{\gamma}\left(m_{1}\right)+q_{1}(t) \Phi_{\gamma}\left(m_{1} \eta_{1}\right)-q_{2}(t)+s_{1}(t) \Phi_{\gamma}\left(m_{1}\right)-s_{2}(t)\right] } \\
& \times \Phi_{\gamma}\left(z_{1}^{\sigma}(t)\right) \\
= & {\left[p(t) z_{1}^{\Delta}(t)\right]^{\Delta}+H\left(t, z_{1}^{\sigma}(t)\right) . }
\end{aligned}
$$

On the other hand, we can also obtain (3.3) if (2.2) is replaced by (2.3). 
Applying Theorem 2.1 with $\alpha(t) \equiv z_{1}(T) \leq z_{1}(t) \equiv \beta(t)$, the equation

$$
\left(p(t) z^{\Delta}(t)\right)^{\Delta}+H\left(t, z^{\sigma}(t)\right)=0, \quad z(T)=z_{1}(T),
$$

has a solution $z(t)$ with $z_{1}(T) \leq z(t) \leq z_{1}(t)$ on $[T, \infty)_{\mathbb{T}}$.

It follows from Lemma 2.1 that $p(t) z^{\Delta}(t)$ is nonincreasing and $\lim _{t \rightarrow \infty} p(t) z^{\Delta}(t):=L>0$ exists (being finite). Integration for $T<s<T^{\prime}$ implies

$$
p\left(T^{\prime}\right) z^{\Delta}\left(T^{\prime}\right)-p(s) z^{\Delta}(s)+\int_{s}^{T^{\prime}} H\left(u, z^{\sigma}(u)\right) \Delta u=0 .
$$

Letting $T^{\prime} \rightarrow \infty$, we obtain

$$
p(s) z^{\Delta}(s)=L+\int_{s}^{\infty} H\left(u, z^{\sigma}(u)\right) \Delta u \geq \int_{s}^{\infty} H\left(u, z^{\sigma}(u)\right) \Delta u .
$$

It follows that

$$
z^{\Delta}(s) \geq \frac{1}{p(s)} \int_{s}^{\infty} H\left(u, z^{\sigma}(u)\right) \Delta u .
$$

Integrating again for $T \leq t_{1}<t$ and by change of integration order [11, Lemma 1]

$$
\int_{s}^{t}\left[\int_{\eta}^{t} f_{1}(\eta, \xi) \Delta \xi\right] \Delta \eta=\int_{s}^{t}\left[\int_{s}^{\sigma(\xi)} f_{1}(\eta, \xi) \Delta \eta\right] \Delta \xi,
$$

we obtain

$$
\begin{aligned}
z(t)-z\left(t_{1}\right) & \geq \int_{t_{1}}^{t} \frac{1}{p(s)} \int_{s}^{\infty} H\left(u, z^{\sigma}(u)\right) \Delta u \Delta s \geq \int_{t_{1}}^{t} \int_{s}^{t} \frac{H\left(u, z^{\sigma}(u)\right)}{p(s)} \Delta u \Delta s \\
& =\int_{t_{1}}^{t} \int_{t_{1}}^{\sigma(u)} \frac{H\left(u, z^{\sigma}(u)\right)}{p(s)} \Delta s \Delta u \geq \int_{t_{1}}^{t} P\left(u, t_{1}\right) H\left(u, z^{\sigma}(u)\right) \Delta u .
\end{aligned}
$$

Consequently, for $t \geq t_{1} \geq T$, we obtain

$$
z(t)-z\left(t_{1}\right) \geq \int_{t_{1}}^{t} P\left(u, t_{1}\right) H\left(u, z^{\sigma}(u)\right) \Delta u .
$$

Since $z(t)$ is bounded and $\int_{t_{1}}^{t} P(u, t) H\left(u, z^{\sigma}(u)\right) \Delta u$ is an increasing function of $t$, it follows that

$$
\int_{t_{1}}^{\infty} P\left(u, t_{1}\right) H\left(u, z^{\sigma}(u)\right) \Delta u<\infty
$$

Let $\alpha=z_{1}^{\sigma}(T)$; by (3.3) and $\Phi_{\gamma}\left(z_{1}^{\sigma}(t)\right)$ being nondecreasing, we have

$$
\int^{\infty} P(u, T) h\left(u, m_{1} \alpha, m_{1} \eta_{1} \alpha, \alpha, m_{1} \alpha, \alpha\right) \Delta u<\infty
$$

So we obtain a contradiction to (3.1). 
Under the assumptions of Theorem 3.1, noting that (3.3), it is easy to obtain the following corollary.

Corollary 3.1 Assume conditions (B1)-(B6) and one of (2.2)-(2.3) hold. Then any bounded solution of $(1.1)$ is oscillatory in the case

$$
\left|\int^{\infty} P(t, a) h\left(t, 0, m_{1} \eta_{1}(t, a) \alpha, 0,0,0\right) \Delta t\right|=\infty
$$

or

$$
\left|\int^{\infty} P(t, a) q_{1}(t) \Phi_{\gamma}\left(m_{1} \eta_{1}(t, a) \alpha\right) \Delta t\right|=\infty .
$$

In order to extend Theorem 3.1 to unbounded solutions, we introduce the class $\Phi$ of functions $\phi$ such that $\phi(u)$ is a nondecreasing continuous function of $u$ satisfying $u \phi(u)>$ $0, u \neq 0$ with

$$
\int_{ \pm u_{0}}^{ \pm \infty} \frac{d u}{\phi(u)}<\infty, \quad u_{0} \neq 0
$$

We say that $h\left(t, u, v_{1}, v_{2}, v_{3}, v_{4}\right)$ satisfies condition $\left(C_{1}\right)$ provided for some $\phi \in \Phi$ there exists $c \neq 0$ such that for all $t \geq T, \eta_{1}=\eta_{1}(t, T)$,

$$
\inf _{|u| \geq|c|} \frac{h\left(t, m_{1} u, m_{1} \eta_{1} u, u, m_{1} u, u\right)}{\phi(u)} \geq k\left|h\left(t, m_{1} c, m_{1} \eta_{1} c, c, m_{1} c, c\right)\right|
$$

for some positive constant $k, m_{1}=1-r_{0}$.

Theorem 3.2 Suppose $\phi \in \Phi$ and $\gamma>1$. Assume (B1)-(B6), and one of (2.2)-(2.3) hold. Furthermore, suppose that the function $h$ satisfies condition $\left(\mathrm{C}_{1}\right)$. If

$$
\left|\int^{\infty} P(t, a) h\left(t, m_{1} \alpha, m_{1} \eta_{1} \alpha, \alpha, m_{1} \alpha, \alpha\right) \Delta t\right|=\infty
$$

holds for all $\alpha \neq 0$ and some sufficiently large $a \in \mathbb{T}$, where $m_{1}=1-r_{0}, \eta_{1}=\eta_{1}(t, a)$ is given in (2.1), then all solutions of (1.1) are oscillatory.

Proof Assume (3.6) holds for all $\alpha \neq 0$ and let $u(t)$ be an eventually positive solution of (1.1) with

$$
u(t)>0, \quad u\left(\tau_{i}(t)\right)>0, \quad u\left(\xi_{j}(t)\right)>0, \quad t \geq T \geq t_{0}, 1 \leq i \leq 2,1 \leq j \leq 2 .
$$

As the proof of Theorem 3.1, we have

$$
z^{\Delta}(s) \geq \frac{1}{p(s)} \int_{s}^{\infty} H\left(u, z^{\sigma}(u)\right) \Delta u,
$$

where $H\left(u, z^{\sigma}(u)\right)=h\left(u, m_{1} z^{\sigma}(u), m_{1} \eta_{1} z^{\sigma}(u), z^{\sigma}(u), m_{1} z^{\sigma}(u), z^{\sigma}(u)\right)$. 
Let $\phi(u)=\Phi_{\gamma}(u)$, then $\phi(u) \in \Phi$. Now multiplying above inequality by $\left[\phi\left(z^{\sigma}(s)\right)\right]^{-1}$, we get

$$
\frac{z^{\Delta}(s)}{\phi\left(z^{\sigma}(s)\right)} \geq \frac{1}{p(s) \phi\left(z^{\sigma}(s)\right)} \int_{s}^{\infty} H\left(u, z^{\sigma}(u)\right) \Delta u=\int_{s}^{\infty} \frac{H\left(u, z^{\sigma}(u)\right)}{p(s) \phi\left(z^{\sigma}(s)\right)} \Delta u .
$$

We next define the continuously differentiable real-valued function

$$
G(u):=\int_{u_{0}}^{u} \frac{d s}{\phi(s)} .
$$

Observe that $G^{\prime}(u)=1 / \phi(u)$. By the Pötzsche chain rule [2, Theorem 1.90],

$$
(G(z(t)))^{\Delta}=\left(\int_{0}^{1} \frac{d h}{\phi\left(z_{h}(t)\right)}\right) z^{\Delta}(t) \geq\left(\int_{0}^{1} \frac{d h}{\phi\left(z^{\sigma}(t)\right)}\right) z^{\Delta}(t)=\frac{z^{\Delta}(t)}{\phi\left(z^{\sigma}(t)\right)},
$$

where $z_{h}(t):=z(t)+h \mu(t) z^{\Delta}(t) \leq z^{\sigma}(t)$.

We claim that

$$
\int_{t_{1}}^{t} \frac{z^{\Delta}(s)}{\phi\left(z^{\sigma}(s)\right)} \Delta s \geq \int_{t_{1}}^{t} \int_{s}^{\infty} \frac{H\left(u, z^{\sigma}(u)\right)}{p(s) \phi\left(z^{\sigma}(s)\right)} \Delta u \Delta s \geq \int_{t_{1}}^{t} P\left(u, t_{1}\right) \frac{H\left(u, z^{\sigma}(u)\right)}{\phi\left(z^{\sigma}(u)\right)} \Delta u
$$

for $t \geq t_{1} \geq T$. By change of integration order [11, Lemma 1], we obtain

$$
\begin{aligned}
\int_{t_{1}}^{t} P\left(u, t_{1}\right) \frac{H\left(u, z^{\sigma}(u)\right)}{\phi\left(z^{\sigma}(u)\right)} \Delta u & =\int_{t_{1}}^{t}\left[\int_{t_{1}}^{u} \frac{1}{p(s)} \Delta s\right] \frac{H\left(u, z^{\sigma}(u)\right)}{\phi\left(z^{\sigma}(u)\right)} \Delta u \\
& \leq \int_{t_{1}}^{t} \int_{t_{1}}^{\sigma(u)} \frac{1}{p(s)} \frac{H\left(u, z^{\sigma}(u)\right)}{\phi\left(z^{\sigma}(u)\right)} \Delta s \Delta u \\
& =\int_{t_{1}}^{t} \int_{s}^{t} \frac{H\left(u, z^{\sigma}(u)\right)}{\phi\left(z^{\sigma}(u)\right)} \frac{1}{p(s)} \Delta u \Delta s \\
& \leq \int_{t_{1}}^{t} \int_{s}^{t} \frac{H\left(u, z^{\sigma}(u)\right)}{p(s) \phi\left(z^{\sigma}(s)\right)} \Delta u \Delta s .
\end{aligned}
$$

For sufficiently large $t_{1}=T$, by condition $\left(\mathrm{C}_{1}\right)$, it immediately follows that

$$
\int_{t_{1}}^{t} \frac{z^{\Delta}(s)}{\phi\left(z^{\sigma}(s)\right)} \Delta s \geq \int_{t_{1}}^{t} P\left(u, t_{1}\right) \frac{H\left(u, z^{\sigma}(u)\right)}{\phi\left(z^{\sigma}(u)\right)} \Delta u \geq k \int_{t_{1}}^{t} P\left(u, t_{1}\right) H(u, c) \Delta u,
$$

where $c:=u(T)+r(T) u(g(T))>0$. Since $\lim _{t \rightarrow \infty} u(t)=\infty$, we have

$$
\lim _{t \rightarrow \infty} G(z(t))=\lim _{t \rightarrow \infty} \int_{z(T)}^{z(t)} \frac{d u}{\phi(u)}=\int_{z(T)}^{\infty} \frac{d u}{\phi(u)}<\infty
$$

by assumption. Therefore,

$$
\int_{z(T)}^{t}(G(z(s)))^{\Delta} \Delta s \geq \int_{z(T)}^{t} \frac{z^{\Delta}(s)}{\phi\left(z^{\sigma}(s)\right)} \Delta s \geq \int_{z(T)}^{t} k P\left(u, t_{1}\right) \frac{H\left(u, z^{\sigma}(u)\right)}{\phi\left(z^{\sigma}(u)\right)} \Delta u .
$$

However, letting $t \rightarrow \infty$ in above inequality, the left side is bounded whereas the right side is unbounded by assumptions (3.6), (3.7). This contradiction shows that (3.6) is sufficient for all solutions of (1.1) to be oscillatory. 
Next we will give two sufficient and necessary conditions for (1.1) being oscillatory under the case $\gamma>1$. However, we need a sufficient condition for (1.1) having a bounded nonoscillatory solution.

Theorem 3.3 Assume conditions (B1)-(B6) hold, and (2.2) or (2.3) holds. If

$$
\int_{a}^{\infty} P(\sigma(t), a) h(t, \alpha, \alpha, 0, \alpha, 0) \Delta t<\infty
$$

for some $\alpha \neq 0$ and some sufficiently large $a \in \mathbb{T}$, then (1.1) has a bounded nonoscillatory solution.

Proof Let

$$
h_{1}(t, \alpha):=h(t, \alpha, \alpha, 0, \alpha, 0) .
$$

Assume that (3.8) holds. Without loss of generality, we may assume $\alpha>0$. Since $p(t)>0$ and $h_{1}(t, \alpha)>0$ on $\mathbb{T}$, by change of integration order [11, Lemma 1], we obtain

$$
\begin{aligned}
\int_{a}^{\infty} \int_{s}^{\infty} \frac{h_{1}(t, \alpha)}{p(s)} \Delta t \Delta s & =\lim _{u \rightarrow+\infty} \int_{a}^{u} \int_{a}^{\sigma(t)} \frac{h_{1}(t, \alpha)}{p(s)} \Delta s \Delta t \\
& =\int_{a}^{\infty} \int_{a}^{\sigma(t)} \frac{h_{1}(t, \alpha)}{p(s)} \Delta s \Delta t \\
& =\int_{a}^{\infty}\left[\int_{a}^{\sigma(t)} \frac{1}{p(s)} \Delta s\right] h_{1}(t, \alpha) \Delta t \\
& =\int_{a}^{\infty} P(\sigma(t), a) h_{1}(t, \alpha) \Delta t<\infty .
\end{aligned}
$$

By (B2) and (3.9), we can choose $T_{0} \in \mathbb{T}$ large enough such that

$$
\int_{T_{0}}^{\infty} \int_{s}^{\infty} \frac{h_{1}(t, \alpha)}{p(s)} \Delta t \Delta s \leq \frac{\left(1-r_{0}\right) \alpha}{2}=\frac{m_{1} \alpha}{2} .
$$

According to (B3) and (B4), we see that there exists $T_{1} \in \mathbb{T}$ with $T_{1}>T_{0}$ such that $g(t) \geq T_{0}$ and $\tau_{i}(t) \geq T_{0}, 1 \leq i \leq 2$, for $t \in\left[T_{1}, \infty\right)_{\mathbb{T}}$.

Define the Banach space $B C\left[T_{0}, \infty\right)_{\mathbb{T}}$ as in (2.8), and let

$$
\Omega=\left\{x=x(t) \in B C\left[T_{0}, \infty\right)_{\mathbb{T}}: \frac{m_{1} \alpha}{2} \leq x(t) \leq \alpha\right\} .
$$

It is easy to verify that $\Omega$ is a bounded, convex, and closed subset of $B C\left[T_{0}, \infty\right)_{\mathbb{T}}$. For the sake of convenience, set

$$
H^{\prime}(t):=h\left(t, x^{\sigma}(t), x\left(\tau_{1}(t)\right), x\left(\tau_{2}(t)\right), x\left(\xi_{1}(t)\right), x\left(\xi_{2}(t)\right)\right) .
$$

For any $x \in \Omega$ and $t \in\left[T_{0}, \infty\right)_{\mathbb{T}}$, we have

$$
0<h(t, \alpha, \alpha, \alpha, \alpha, \alpha)<H^{\prime}(t) \leq h\left(t, \alpha, \alpha, m_{1} \alpha / 2, \alpha, m_{1} \alpha / 2\right) \leq h_{1}(t, \alpha) .
$$


Now we define two operators $U$ and $S: \Omega \rightarrow B C\left[T_{0}, \infty\right)_{\mathbb{T}}$ as follows:

$$
(U x)(t)= \begin{cases}-r(t) x(g(t)), & t \in\left[T_{1}, \infty\right)_{\mathbb{T}}, \\ (U x)\left(T_{1}\right), & t \in\left[T_{0}, T_{1}\right]_{\mathbb{T}}\end{cases}
$$

and

$$
(S x)(t)= \begin{cases}\alpha-\int_{t}^{\infty} \int_{s}^{\infty} \frac{H^{\prime}(u)}{p(s)} \Delta u \Delta s, & t \in\left[T_{1}, \infty\right)_{\mathbb{T}}, \\ \alpha-\int_{T_{1}}^{\infty} \int_{s}^{\infty} \frac{H^{\prime}(u)}{p(s)} \Delta u \Delta s, & t \in\left[T_{0}, T_{1}\right]_{\mathbb{T}} .\end{cases}
$$

Next, we will show that $U$ and $S$ satisfy the conditions in Lemma 2.3.

(i) We first prove that $U x+S y \in \Omega$ for any $x, y \in \Omega$. Note that for any $x, y \in \Omega, m_{1} \alpha / 2 \leq$ $x, y \leq \alpha$. For any $x, y \in \Omega$ and $t \in\left[T_{1}, \infty\right)_{\mathbb{T}}$, by (3.10)-(3.11), we have

$$
(U x)(t)+(S y)(t)=\alpha-r(t) x(g(t))-\int_{t}^{\infty} \int_{s}^{\infty} \frac{H^{\prime}(u)}{p(s)} \Delta u \Delta s \geq m_{1} \alpha-\frac{m_{1} \alpha}{2} \geq \frac{m_{1} \alpha}{2}
$$

and

$$
(U x)(t)+(S y)(t)=\alpha-r(t) x(g(t))-\int_{t}^{\infty} \int_{s}^{\infty} \frac{H^{\prime}(u)}{p(s)} \Delta u \Delta s \leq \alpha .
$$

Similarly, we can show that $m_{1} \alpha / 2 \leq U x+S y \leq \alpha$ for any $x, y \in \Omega$ and $t \in\left[T_{0}, T_{1}\right]_{\mathbb{T}}$.

(ii) It is not difficult to check that $U$ is a contraction mapping.

(iii) We will prove that $S$ is a completely continuous mapping. It is easy to check that $S$ maps $\Omega$ into $\Omega$.

Again, for the sake of convenience, let

$$
H_{l}(t):=h\left(t, x_{l}^{\sigma}(t), x_{l}\left(\tau_{1}(t)\right), x_{l}\left(\tau_{2}(t)\right), x_{l}\left(\xi_{1}(t)\right), x_{l}\left(\xi_{2}(t)\right)\right) .
$$

Next, we show that the continuity of $S$. Let $x_{l} \in \Omega$ and $\left\|x_{l}-x\right\| \rightarrow 0$ as $l \rightarrow \infty$, then $x \in \Omega$ and $x_{l} \rightarrow x$ as $l \rightarrow \infty$. By the monotonicity and continuity of $h$, as $l \rightarrow \infty$, we have

$$
\left\{\begin{array}{l}
\left|H_{l}(t)-H^{\prime}(t)\right| \rightarrow 0, \\
\left|H_{l}(t)-H^{\prime}(t)\right| \leq 2 h_{1}(t, \alpha) .
\end{array}\right.
$$

For $t \in\left[T_{1}, \infty\right)_{\mathbb{T}}$, we have

$$
\begin{aligned}
\left\|S x_{l}-S x\right\| & =\left|S x_{l}-S x\right| \\
& \leq \int_{t}^{\infty} \int_{s}^{\infty} \frac{\left|H_{l}(u)-H^{\prime}(u)\right|}{p(s)} \Delta u \Delta s
\end{aligned}
$$

and $\left|\left(S x_{l}\right)(t)-(S x)(t)\right|=0$ for $t \in\left[T_{0}, T_{1}\right]_{\mathbb{T}}$. Employing Lebesgue's dominated convergence theorem [3, Chapter 5], we get

$$
\left\|S x_{l}-S x\right\|=\left|S x_{l}-S x\right| \rightarrow 0 \quad \text { as } l \rightarrow \infty .
$$

Thus $S$ is continuous. 
Third, we show $S \Omega$ is relatively compact. According to Lemma 2.2, it suffices to show that $S \Omega$ is bounded, uniformly Cauchy, and equi-continuous. The boundedness is obvious.

For any $x \in \Omega$, by (3.10)-(3.11), we have

$$
\int_{T_{1}}^{\infty} \int_{s}^{\infty} \frac{H^{\prime}(u)}{p(s)} \Delta u \Delta s<\infty
$$

Then for any given $\varepsilon>0$, there exists $T_{2} \in\left[T_{1}, \infty\right)_{\mathbb{T}}$ large enough such that

$$
\int_{T_{2}}^{\infty} \int_{s}^{\infty} \frac{H^{\prime}(u)}{p(s)} \Delta u \Delta s<\varepsilon / 2 .
$$

Hence, for any $x \in \Omega$ and $t_{1}, t_{2} \in\left[T_{2}, \infty\right)_{\mathbb{T}}$, we have

$$
\begin{aligned}
\left\|(S x)\left(t_{1}\right)-(S x)\left(t_{2}\right)\right\| & =\left|(S x)\left(t_{1}\right)-(S x)\left(t_{2}\right)\right| \\
& =\left|\int_{t_{1}}^{\infty} \int_{s}^{\infty} \frac{H^{\prime}(u)}{p(s)} \Delta u \Delta s-\int_{t_{2}}^{\infty} \int_{s}^{\infty} \frac{H^{\prime}(u)}{p(s)} \Delta u \Delta s\right| \\
& \leq 2 \int_{T_{2}}^{\infty} \int_{s}^{\infty} \frac{H^{\prime}(u)}{p(s)} \Delta u \Delta s \\
& <2 \times \frac{\varepsilon}{2}=\varepsilon .
\end{aligned}
$$

So $S \Omega$ is uniformly Cauchy.

Finally, we will prove that $S \Omega$ is equi-continuous. For $T_{1} \leq t_{1}<t_{2} \leq T_{2}+1$, we have

$$
\begin{aligned}
\left\|(S x)\left(t_{1}\right)-(S x)\left(t_{2}\right)\right\| & =\left|(S x)\left(t_{1}\right)-(S x)\left(t_{2}\right)\right| \\
& =\left|\int_{t_{1}}^{\infty} \int_{s}^{\infty} \frac{H^{\prime}(u)}{p(s)} \Delta u \Delta s-\int_{t_{2}}^{\infty} \int_{s}^{\infty} \frac{H^{\prime}(u)}{p(s)} \Delta u \Delta s\right| \\
& \leq \int_{t_{1}}^{t_{2}} \int_{s}^{\infty} \frac{H^{\prime}(u)}{p(s)} \Delta u \Delta s .
\end{aligned}
$$

For $t_{1}<T_{1} \leq t_{2} \leq T_{2}+1$, we have

$$
\left\|(S x)\left(t_{1}\right)-(S x)\left(t_{2}\right)\right\|=\left|(S x)\left(t_{1}\right)-(S x)\left(t_{2}\right)\right| \leq \int_{T_{1}}^{t_{2}} \int_{s}^{\infty} \frac{H^{\prime}(u)}{p(s)} \Delta u \Delta s .
$$

For $t_{1}, t_{2} \in\left[T_{1}, T_{2}\right]_{\mathbb{T}},\left\|(S x)\left(t_{1}\right)-(S x)\left(t_{2}\right)\right\|=0$.

Therefore there exists $0<\delta<1$ such that $\left|(S x)\left(t_{1}\right)-(S x)\left(t_{2}\right)\right|<\varepsilon$ if $t_{1}, t_{2} \in\left[T_{0}, T_{2}+1\right)$ and $\left|t_{2}-t_{1}\right|<\delta$. This means that $S \Omega$ is equi-continuous.

It follows from Lemma 2.2 that $S \Omega$ is relatively compact, and then $S$ is completely continuous.

By Lemma 2.3, there exists $x \in \Omega$ such that $(U+S) x=x$, which indicates that $x(t)$ is a solution of (1.1). In particular, for $t \in\left[T_{1}, \infty\right)_{\mathbb{T}}$, we have

$$
x(t)=\alpha-r(t) x(g(t))-\int_{t}^{\infty} \int_{s}^{\infty} \frac{H^{\prime}(u)}{p(s)} \Delta u \Delta s .
$$

Let $t \rightarrow \infty$, we obtain the desired result. 
Remark 3.1 Similar to the proof of Theorem 3.3, under the assumptions of Theorem 3.3, if

$$
\int_{a}^{\infty} P(\sigma(t), a) h\left(t, \alpha, \alpha, \frac{\left(1-r_{0}\right) \alpha}{2}, \alpha, \frac{\left(1-r_{0}\right) \alpha}{2}\right) \Delta t<\infty
$$

for some $\alpha \neq 0$, then (1.1) has a bounded nonoscillatory solution.

Theorem 3.3 plays an important role in excluding (1.1) to have a unbounded nonoscillatory solution under that (3.8) holds.

Theorem 3.4 Suppose $\phi \in \Phi$ and $\gamma>1$. Assume (B1)-(B6) and one of (2.2)-(2.3) hold, and the function $h$ satisfies condition $\left(C_{1}\right)$. Furthermore, suppose that there exists $\rho_{1}>0$ such that

$$
\liminf _{t \rightarrow \infty} \eta_{1}(t, a) \geq \rho_{1} \quad \text { for } a \in \mathbb{T}
$$

and

$$
\left|\int^{\infty} P(t, T) h(t, 0,0, \alpha, 0, \alpha) \Delta t\right|<\infty \quad \text { for all } \alpha \neq 0 .
$$

Then a sufficient and necessary condition for (1.1) to be oscillatory is that

$$
\left|\int^{\infty} P(t, a) h\left(t, \alpha, \alpha \eta_{1}, 0, \alpha, 0\right) \Delta t\right|=\infty
$$

holds for all $\alpha \neq 0$ and some sufficiently large $a \in \mathbb{T}$, where $\eta_{1}=\eta_{1}(t, a)$ is given in (2.1).

Proof Assume that (3.14) holds for all $\alpha \neq 0$ and let $u(t)>0$ be a nonoscillatory solution of (1.1). Similar to the proof of Theorem 3.2, $c=u(T)+r(T) u(g(T))>0$, we obtain (3.7):

$$
\int_{t_{1}}^{t} \frac{z^{\Delta}(s)}{\phi\left(z^{\sigma}(s)\right)} \Delta s \geq \int_{t_{1}}^{t} P\left(u, t_{1}\right) \frac{H\left(u, z^{\sigma}(u)\right)}{\phi\left(z^{\sigma}(u)\right)} \Delta u \geq k \int_{t_{1}}^{t} P\left(u, t_{1}\right) H(u, c) \Delta u .
$$

By (3.12)-(3.14), for sufficiently large $t \gg t_{1} \geq T$, we have

$$
\begin{aligned}
\int_{t_{1}}^{t} P\left(u, t_{1}\right) H(u, c) \Delta u= & \int_{t_{1}}^{t} P\left(u, t_{1}\right) h\left(u, m_{1} c, m_{1} \eta_{1} c, c, m_{1} c, c\right) \Delta u \\
= & \int_{t_{1}}^{t} P\left(u, t_{1}\right) h\left(u, m_{1} c, m_{1} \eta_{1} c, 0, m_{1} c, 0\right) \Delta u \\
& +\int_{t_{1}}^{t} P\left(u, t_{1}\right) h(u, 0,0, c, 0, c) \Delta u \\
& \sim \int_{t_{1}}^{t} P\left(u, t_{1}\right) h\left(u, m_{1} c, m_{1} \eta_{1} c, 0, m_{1} c, 0\right) \Delta u \\
& =\int_{t_{1}}^{t} P\left(u, t_{1}\right) h\left(u, \alpha, \alpha \eta_{1}, 0, \alpha, 0\right) \Delta u,
\end{aligned}
$$


where $\alpha=m_{1} c$. The rest of the proof is the same as Theorem 3.2. So we leave details to readers.

Conversely, assume that (3.12)-(3.13) hold and (3.14) does not hold for some $\alpha \neq 0$, then we have

$$
\left|\int^{\infty} P(t, a) h\left(t, \alpha, \alpha \eta_{1}(t, a), 0, \alpha, 0\right) \Delta t\right|<\infty .
$$

Note that (3.12) implies that $P(\sigma(t), a) / P(t, a)$ is bounded on $\mathbb{T}$, and (3.15) holds if and only if

$$
\left|\int^{\infty} P(\sigma(t), a) h\left(t, \alpha, \alpha \eta_{1}(t, a), 0, \alpha, 0\right) \Delta t\right|<\infty .
$$

According to (3.12), it follows that for any $\varepsilon>0$ with $\varepsilon<\frac{1}{2} \min \rho_{1}$, there exists $T_{1} \geq t_{0}$ such that $\eta_{1}(t, a) \geq \rho_{1}-\varepsilon=: \hat{\rho}>0$ for $t \geq T_{1}$. It follows that $\left|\alpha \eta_{1}(t, a)\right| \geq|\alpha \hat{\rho}|$ for $t \geq T_{1}$. Then by the monotonicity of $h$ and the fact that $\eta_{1}(t, a) \leq 1$ for $t \geq T_{1}$, we have

$$
\left|\int_{a}^{\infty} P(\sigma(t), a) h(t, \alpha \hat{\rho}, \alpha \hat{\rho}, 0, \alpha \hat{\rho}, 0) \Delta t\right|<\infty
$$

which gives (3.8). Therefore, by Theorem 3.3, equation (1.1) has a bounded nonoscillatory solution. This contradiction shows that (3.14) is necessary.

If (3.13) is false, (3.14) is not a necessary condition for (1.1) being oscillatory. Suppose that there exist constants $\beta_{1}>1, \beta_{2}>1, \beta_{3}>1$ such that

$$
q_{0}(t) \Phi_{\gamma}\left(m_{1}\right)+q_{1}(t) \Phi_{\gamma}\left(m_{1} \eta_{1}\right)>\beta_{1} q_{2}(t), \quad s_{1}(t) \Phi_{\gamma}\left(m_{1}\right)>\beta_{2} s_{2}(t)
$$

and

$$
q_{0}(t) \Phi_{\gamma}\left(m_{1}\right)+q_{1}(t) \Phi_{\gamma}\left(m_{1} \eta_{1}\right)+s_{1}(t) \Phi_{\gamma}\left(m_{1}\right)>\beta_{3}\left[q_{2}(t)+s_{2}(t)\right] .
$$

We give another sufficient and necessary condition for (1.1) to be oscillatory.

Theorem 3.5 Suppose $\phi \in \Phi$ and $\gamma>1$. Assume (B1)-(B6) and one of (2.2)-(2.3) hold, and the function $h$ satisfies condition $\left(\mathrm{C}_{1}\right)$. Furthermore, suppose that (3.12) and one of (3.16)(3.17) hold. Then a sufficient and necessary condition for the second order nonlinear neutral dynamic equation (1.1) to be oscillatory is that

$$
\left|\int^{\infty} P(t, a) h\left(t, \alpha, \alpha \eta_{1}, 0, \alpha, 0\right) \Delta t\right|=\infty
$$

holds for all $\alpha \neq 0$ and some sufficiently large $a \in \mathbb{T}$, where $\eta_{1}=\eta_{1}(t, a)$ is given in (2.1).

Since the proof of Theorem 3.5 is similar to that of Theorem 3.4, we leave the details to the readers.

For the case $0<\gamma \leq 1$, Theorems 3.2, 3.4, and 3.5 do not hold. We introduce another class $\Psi$ of functions $\psi$ such that $\psi(u)$ is a nondecreasing continuous function of $u$ satis- 
fying $u \phi(u)>0, u \neq 0$ with

$$
\int_{ \pm u_{0}}^{ \pm \infty} \frac{d u}{\psi(u)}=\infty \quad \text { and } \quad \int_{ \pm u_{0}}^{ \pm \infty} \frac{d u}{\Phi_{\gamma}(u)|\psi(u)|}<\infty, \quad u_{0} \neq 0
$$

Theorem 3.6 Assume $0<\gamma \leq 1$, conditions (B1)-(B6) and one of (2.2)-(2.3) hold. Furthermore, suppose that there exists a function $\psi \in \Psi$ which satisfies (3.19). Then any solution $x(t)$ of $(1.1)$ is oscillatory in the case

$$
\left|\int^{\infty}[P(t, a)]^{\gamma-\varepsilon} h\left(t, m_{1} \alpha, m_{1} \alpha \eta_{1}, \alpha, m_{1} \alpha, \alpha\right) \Delta t\right|=\infty
$$

for all $\alpha \neq 0$, some sufficiently large $a \in \mathbb{T}$, and $\forall 0<\varepsilon \leq \gamma$, where $\eta_{1}=\eta_{1}(t, a)$ is given in (2.1).

Proof Assume not and let $u(t)$ be a bounded nonoscillatory solution of (1.1) which we may assume satisfies

$$
u(t)>0, \quad u\left(\tau_{i}(t)\right)>0, \quad u\left(\xi_{j}(t)\right)>0, \quad t \geq T \geq t_{0}, 1 \leq i \leq 2,1 \leq j \leq 2 .
$$

Let

$$
Q(t)=q_{0}(t) \Phi_{\gamma}\left(m_{1}\right)+q_{1}(t) \Phi_{\gamma}\left(m_{1} \eta_{1}\right)-q_{2}(t)+s_{1}(t) \Phi_{\gamma}\left(m_{1}\right)-s_{2}(t) .
$$

As the proof of Theorem 3.1, we also get (3.3), i.e.

$$
\begin{aligned}
& {\left[p(t) z_{1}^{\Delta}(t)\right]^{\Delta}+\left[\left(q_{0}(t)+q_{1}(t) \Phi_{\gamma}\left(\eta_{1}\right)+s_{1}(t)\right) \Phi_{\gamma}\left(m_{1}\right)-q_{2}(t)-s_{2}(t)\right] \Phi_{\gamma}\left(z_{1}^{\sigma}(t)\right)} \\
& \quad=\left[p(t) z_{1}^{\Delta}(t)\right]^{\Delta}+H\left(t, z_{1}^{\sigma}(t)\right) \\
& \quad=\left[p(t) z_{1}^{\Delta}(t)\right]^{\Delta}+Q(t) \Phi_{\gamma}\left(z_{1}^{\sigma}(t)\right) \leq 0 .
\end{aligned}
$$

By Lemma 2.1, $p(t) z_{1}^{\Delta}(t) \leq p\left(T_{1}\right) z_{1}^{\Delta}\left(T_{1}\right)=c_{1}>0$ for $t \geq T_{1}$. Integrating it from $T_{1}$ to $t$, for $t>T_{2}>T_{1}$, we have

$$
\begin{aligned}
z_{1}(t) & \leq z_{1}\left(T_{1}\right)+c_{1} \int_{T_{1}}^{t} \frac{\Delta s}{p(s)} \leq\left[\frac{z_{1}\left(T_{1}\right)}{P\left(t, T_{1}\right)}+c_{1}\right] P\left(t, T_{1}\right) \\
& \leq\left[\frac{z_{1}\left(T_{1}\right)}{P\left(T_{2}, T_{1}\right)}+c_{1}\right] P\left(t, T_{1}\right)=c_{2} P\left(t, T_{1}\right) .
\end{aligned}
$$

Let

$$
\begin{aligned}
& \psi(u)=\Phi_{1-\gamma+\varepsilon}(u), \quad Q_{1}(t)=Q(t) /\left|\psi\left(z_{1}(t) / c_{2}\right)\right|, \\
& H_{1}\left(z_{1}(t)\right)=\Phi_{\gamma}\left(z_{1}(t)\right)\left|\psi\left(z_{1}(t) / c_{2}\right)\right|, \quad \forall 1>\varepsilon>0 .
\end{aligned}
$$

By (3.21) and (3.22), we obtain

$$
\left[p(t) z_{1}^{\Delta}(t)\right]^{\Delta}+Q_{1}(t) H_{1}\left(z_{1}(t)\right) \leq\left[p(t) z_{1}^{\Delta}(t)\right]^{\Delta}+Q(t) \Phi_{\gamma}\left(z_{1}^{\sigma}(t)\right) \leq 0 .
$$


Integrating the above inequality from $t$ to $T_{3}, T_{1} \leq t \leq T_{3}$, we have

$$
z_{1}^{\Delta}(t) \geq \frac{1}{p(t)} \int_{t}^{T_{3}} Q_{1}(s) H_{1}\left(z_{1}(s)\right) \Delta s
$$

Then, similar to the proof of Theorem 3.2, we have

$$
\begin{aligned}
\lim _{T_{3} \rightarrow \infty} \int_{T_{1}}^{T_{3}} \frac{z_{1}^{\Delta}(t) \Delta t}{H_{1}\left(z_{1}(t)\right)} & =\lim _{T_{3} \rightarrow \infty} \int_{z_{1}\left(T_{1}\right)}^{z_{1}\left(T_{3}\right)} \frac{\Delta u}{H_{1}(u)} \geq \lim _{T_{3} \rightarrow \infty} \int_{T_{1}}^{T_{3}} P\left(\sigma(s), T_{1}\right) Q_{1}(s) \Delta s \\
& =\int_{T_{1}}^{\infty} P\left(\sigma(s), T_{1}\right) Q_{1}(s) \Delta s \geq \int_{T_{1}}^{\infty} P\left(s, T_{1}\right) Q_{1}(s) \Delta s .
\end{aligned}
$$

According to (3.19), we have

$$
\int_{ \pm u_{0}}^{ \pm \infty} \frac{d u}{H_{1}(u)}=\int_{ \pm u_{0}}^{ \pm \infty} \frac{d u}{\Phi_{\gamma}(u)|\psi(u)|}<\infty, \quad u_{0} \neq 0 .
$$

However, by (3.20) and (3.22), we have

$$
\int_{T_{1}}^{\infty} P\left(s, T_{1}\right) Q_{1}(s) \Delta s \geq \int_{T_{1}}^{\infty} \frac{P\left(s, T_{1}\right) Q(s)}{\left|\psi\left(z_{1}(s) / c_{2}\right)\right|} \Delta s \geq \int_{T_{1}}^{\infty} \frac{P\left(s, T_{1}\right)}{\left|\psi\left(P\left(s, T_{1}\right)\right)\right|} Q(s) \Delta s=\infty,
$$

which contradicts $\int_{T_{1}}^{\infty} P\left(s, T_{1}\right) Q_{1}(s) \Delta s \leq \int_{u_{0}}^{+\infty} \frac{d u}{H_{1}(u)}<\infty$.

We will show that Theorem 3.6 is also true if $\varepsilon=0$ in (3.20) for $0<\gamma<1$.

Let $u, v, t, s \in \mathbb{T}$ with $u, v, t, s \geq T_{0}$. Suppose that $x(t)>0$ be a solution of (1.1). Integrating (1.1) from $v$ to $s$ and dividing the resulting equation by $p(s)$, we have

$$
z^{\Delta}(s)=p(v) z^{\Delta}(v) / p(s)-\frac{1}{p(s)} \int_{v}^{s} h \Delta \theta_{1} .
$$

Again, integrating the above mentioned inequality from $u$ to $t$, by Lemma 2.1 and (3.3), we have

$$
\begin{aligned}
z(t) & =z(u)+p(v) z^{\Delta}(v) P(t, u)+\int_{u}^{t} \frac{1}{p(s)} \int_{s}^{v} h \Delta \theta_{1} \Delta s \\
& \geq \int_{u}^{t} \frac{1}{p(s)} \int_{s}^{v} H\left(\theta_{1}, z^{\sigma}\left(\theta_{1}\right)\right) \Delta \theta_{1} \Delta s .
\end{aligned}
$$

Set $u=T_{0}$ and $v>t \geq s \geq T_{1} \geq T_{0}$, using (3.22) and $H\left(\theta_{1}, z\left(\theta_{1}\right)\right)=\frac{H\left(\theta_{1}, c_{2} P\left(\theta_{1}, T_{0}\right)\right)}{\left[c_{2} P\left(\theta_{1}, T_{0}\right)\right]^{\gamma}}\left(z\left(\theta_{1}\right)\right)^{\gamma}$, we find

$$
\begin{aligned}
z(t) & \geq \int_{u}^{t} \frac{1}{p(s)} \int_{s}^{v} H\left(\theta_{1}, z^{\sigma}\left(\theta_{1}\right)\right) \Delta \theta_{1} \Delta s \geq \int_{T_{0}}^{t} \frac{1}{p(s)} \int_{t}^{v} H\left(\theta_{1}, z^{\sigma}\left(\theta_{1}\right)\right) \Delta \theta_{1} \Delta s \\
& \geq P\left(t, T_{0}\right) \int_{t}^{v} H\left(\theta_{1}, z\left(\theta_{1}\right)\right) \Delta \theta_{1}=P\left(t, T_{0}\right) \int_{t}^{v} \frac{H\left(\theta_{1}, c_{2} P\left(\theta_{1}, T_{0}\right)\right)}{\left[c_{2} P\left(\theta_{1}, T_{0}\right)\right]^{\gamma}}\left(z\left(\theta_{1}\right)\right)^{\gamma} \Delta \theta_{1},
\end{aligned}
$$

and hence

$$
\frac{z(t)}{P\left(t, T_{0}\right)} \geq Z(t), \quad \text { where } Z(t)=\left(c_{2}\right)^{-\gamma} \int_{t}^{\nu} H\left(\theta_{1}, c_{2} P\left(\theta_{1}, T_{0}\right)\right) \frac{\left(z\left(\theta_{1}\right)\right)^{\gamma}}{\left[P\left(\theta_{1}, T_{0}\right)\right]^{\gamma}} \Delta \theta_{1}>0 .
$$


Thus, by [9, Lemma 2.1], we get

$$
\begin{aligned}
-Z^{\Delta}\left(\theta_{1}\right) & \geq\left(c_{2}\right)^{-\gamma} H\left(\theta_{1}, c_{2} P\left(\theta_{1}, T_{0}\right)\right)\left[Z\left(\theta_{1}\right)\right]^{\gamma} \\
& \geq \frac{(1-\gamma)\left(-Z^{\Delta}\left(\theta_{1}\right)\right)}{\left(c_{2}\right)^{\gamma}\left(-\left[Z^{1-\gamma}\right]^{\Delta}\left(\theta_{1}\right)\right)} H\left(\theta_{1}, c_{2} P\left(\theta_{1}, T_{0}\right)\right),
\end{aligned}
$$

which implies

$$
-\left[Z^{1-\gamma}\right]^{\Delta}\left(\theta_{1}\right) \geq \frac{1-\gamma}{\left(c_{2}\right)^{\gamma}} H\left(\theta_{1}, c_{2} P\left(\theta_{1}, T_{0}\right)\right) .
$$

Integrating it from $T_{0}$ to $t$ and letting $t \rightarrow \infty$, we obtain

$$
\begin{aligned}
{\left[Z\left(T_{0}\right)\right]^{1-\gamma} } & \geq[Z(t)]^{1-\gamma}+\frac{1-\gamma}{\left(c_{2}\right)^{\gamma}} \int_{T_{0}}^{t} H\left(\theta_{1}, c_{2} P\left(\theta_{1}, T_{0}\right)\right) \Delta \theta_{1} \\
& \geq \frac{1-\gamma}{\left(c_{2}\right)^{\gamma}} \int_{T_{0}}^{t} H\left(\theta_{1}, c_{2} P\left(\theta_{1}, T_{0}\right)\right) \Delta \theta_{1},
\end{aligned}
$$

which contradicts (3.20) for $\varepsilon=0$.

So we have the following conclusion.

Theorem 3.7 Assume $0<\gamma<1$, conditions (B1)-(B6) and one of (2.2)-(2.3) hold. Then any solution $x(t)$ of $(1.1)$ is oscillatory in the case

$$
\left|\int^{\infty}[P(t, a)]^{\gamma} h\left(t, m_{1} \alpha, m_{1} \alpha \eta_{1}, \alpha, m_{1} \alpha, \alpha\right) \Delta t\right|=\infty
$$

for all $\alpha \neq 0$ and some sufficiently large $a \in \mathbb{T}$, where $\eta_{1}=\eta_{1}(t, a)$ is given in (2.1).

Is Theorem 3.7 also true if $0<\gamma<1$ is replaced by $\gamma>1$ ? In general, it is not true. It is easy to see that (3.23) holds for $\gamma>1$, which does not imply (3.1) for all $\alpha \neq 0$. Then, if (3.12)-(3.13) hold, by Theorem 3.3, all bounded solutions of (1.1) may be nonoscillatory. But it is true for unbounded solutions of (1.1).

Theorem 3.8 Assume $\gamma>1$, conditions (B1)-(B6), and one of (2.2)-(2.3) hold. Then any unbounded solution $x(t)$ of $(1.1)$ is oscillatory in the case

$$
\left|\int^{\infty}[P(t, a)]^{\gamma} h\left(t, m_{1} \alpha, m_{1} \alpha \eta_{1}, \alpha, m_{1} \alpha, \alpha\right) \Delta t\right|=\infty
$$

for all $\alpha \neq 0$ and some sufficiently large $a \in \mathbb{T}$, where $\eta_{1}=\eta_{1}(t, a)$ is given in (2.1).

Proof Assume $x(t)>0$ be an unbounded solution of (1.1). Integrating (1.1) from $T_{1}$ to $t$, using $t \geq T_{1} \geq T_{0}$, and by (B1) and Lemma 2.1, we have

$$
\begin{aligned}
z(t)-z\left(T_{1}\right) & =\int_{T_{1}}^{t} z^{\Delta}(s) \Delta s=\int_{T_{1}}^{t} \frac{p(s) z^{\Delta}(s)}{p(s)} \Delta s \\
& \geq P\left(t, T_{1}\right)\left[p(t) z^{\Delta}(t)\right]-\int_{T_{1}}^{t} P\left(t, T_{1}\right)\left[p(s) z^{\Delta}(s)\right]^{\Delta} \Delta s
\end{aligned}
$$


which implies that

$$
z(t)>P\left(t, T_{1}\right)\left[p(t) z^{\Delta}(t)\right], \quad t \geq T_{1} \geq T_{0} .
$$

By (3.3) and $z^{\Delta}(t)>0$ for $t \geq T_{0}$, we get

$$
H\left(t, z^{\sigma}(t)\right) \geq H(t, z(t))>H\left(t, P\left(t, T_{1}\right)\left[p(t) z^{\Delta}(t)\right]\right)=Q(t) \Phi_{\gamma}\left(P\left(t, T_{1}\right)\left[p(t) z^{\Delta}(t)\right]\right)>0 .
$$

Let $y(t)=p(t) z^{\Delta}(t)$, then

$$
-y^{\Delta}(t) \geq H\left(t, z^{\sigma}(t)\right)>Q(t) \Phi_{\gamma}\left(P\left(t, T_{1}\right) y(t)\right)>0 .
$$

Both sides divide $\Phi_{\gamma}(y(t))$; integrating it from $T_{2}$ to $T_{3}, T_{3}>T_{2} \geq T_{1}$, and noting that $0 \leq y(t)<y\left(T_{0}\right), t \geq T_{0}$, we have

$$
\begin{aligned}
0 & <\int_{T_{2}}^{T_{3}} Q(t) \Phi_{\gamma}\left(P\left(t, T_{1}\right)\right) \Delta t<\int_{T_{2}}^{T_{3}} \frac{-y^{\Delta}(t)}{\Phi_{\gamma}(y(t))} \Delta t \\
& =\int_{y\left(T_{3}\right)}^{y\left(T_{2}\right)} \frac{1}{\Phi_{\gamma}(u)} \Delta u \leq \int_{0}^{y\left(T_{2}\right)} \frac{1}{\Phi_{\gamma}(u)} \Delta u=\infty .
\end{aligned}
$$

Letting $T_{3} \rightarrow \infty$ and $a=T_{1}$, we obtain

$$
0<\int_{T_{2}}^{\infty} Q(t) \Phi_{\gamma}\left(P\left(t, T_{1}\right)\right) \Delta t=\int_{T_{2}}^{\infty}\left[P\left(t, T_{1}\right)\right]^{\gamma} h\left(t, m_{1} \alpha, m_{1} \eta_{1} \alpha, \alpha, m_{1} \alpha, \alpha\right) \Delta t<\infty,
$$

which contradicts (3.24). The proof is complete.

\section{Examples}

We would like to illustrate the results by means of the following examples.

Example 4.1 Let $\Phi_{*}(u)=|u|^{*-1} u, n \in \mathbb{N}, \sigma^{n}(t)=\sigma\left(\sigma^{n-1}(t)\right), \rho^{n}(t)=\rho^{n-1}(t), h>0$ is a constant, time scale $\mathbb{T}=h \mathbb{Z}$. Consider the dynamic equation

$$
\left[p(t)\left(x(t)+r_{0} x(g(t))\right)^{\Delta}\right]^{\Delta}+h\left(\sigma(t), x\left(\tau_{1}(t)\right), x\left(\tau_{2}(t)\right), x\left(\xi_{1}(t)\right), x\left(\xi_{2}(t)\right)\right)=0,
$$

where $g(t)=\rho(t), 0 \leq r_{0}<1$ is a constant. $\tau_{1}(t)=\rho(t), \tau_{2}(t)=t, \xi_{1}(t)=\sigma^{3}(t), \xi_{2}(t)=\sigma^{2}(t)$, $q_{0}(t)=t, q_{1}(t)=\sqrt{t}, q_{2}(t)=\ln t, s_{1}(t)=1, s_{2}(t)=t^{-2}$. We assume that $p(t)>0$ is nondecreasing and satisfies (B1). For sufficiently large $t \in \mathbb{T}$, it is not difficult to check that conditions (B2)-(B6), (2.2), and (3.17) hold. By Lemma 2.1, for sufficiently large $t \in \mathbb{T}$, we have

$$
\eta_{1} \sim \tau_{1}(t) / \sigma(t)=(t-h) /(t+h) \sim 1 \quad \text { and } \quad P(t, a)=\int_{a}^{t} \frac{\Delta s}{p(s)} \geq 1, \quad t \gg a \in \mathbb{T}
$$

and

$$
\left|\int^{\infty} q_{1}(t) \Phi_{\gamma}\left(m_{1} \eta_{1} \alpha\right) \Delta t\right|=\left|\int^{\infty} q_{1}(t) \Phi_{\gamma}\left(m_{1} \alpha\right) \Delta t\right|=\infty .
$$

According to Corollary 3.1, any bounded solution of (4.1) is oscillatory. 
Let $\phi(u)=\Phi_{\gamma}(u)$ and $\gamma>1$, we obtain

$$
\inf _{|u| \geq|c|} \frac{h\left(t, m_{1} u, m_{1} \eta_{1} u, u, m_{1} u, u\right)}{\phi(u)} \geq k\left|h\left(t, m_{1} c, m_{1} \eta_{1} c, c, m_{1} c, c\right)\right|
$$

for some positive constant $k$ and $k\left|\Phi_{\gamma}(c)\right| \leq 1$. Condition $\left(\mathrm{C}_{1}\right)$ is satisfied. Since $\eta_{1} \sim 1$, (3.12) holds. Equations (4.2) and (3.17) imply (3.18) holds, so we give a sufficient and necessary condition for all solutions of (4.1) being oscillatory.

Let $\psi(u)=\Phi_{1-\gamma+\varepsilon}(u), 0<\varepsilon \leq \gamma<1$, then (3.19) holds. Hence, by Theorem 3.6, all solutions of (1.1) are oscillatory because (4.2) and (3.17) imply (3.20).

In particular, (4.1) becomes the classical difference equation if $h=1$.

Remark 4.1 Let $\mathbb{T}=q^{\mathbb{N}}, q>1$ be a constant; $\mathbb{T}=\mathbb{N}_{0}^{2}=\left\{n^{2}: n \in \mathbb{N}_{0}\right\} ; \mathbb{T}=\left\{H_{n}: n \in \mathbb{N}_{0}\right\}$, where $H_{n}$ are the so-called harmonic numbers, $H_{0}=0, H_{n}=\sum_{k=1}^{n} \frac{1}{k}$ for $n \in \mathbb{N}$. Then the conclusion of Example 4.1 is also true under the same assumptions.

Example 4.2 Let $\Phi_{*}(u)=|u|^{*-1} u$, the time scale $\mathbb{T}=\mathbb{P}_{a, b}=\bigcup_{k=0}^{\infty}[k(a+b), k(a+b)+a]$, $a>0, b \geq 0$ are constants. Consider the dynamic equation

$$
\begin{aligned}
& {\left[t^{-3}\left(x(t)+\left(1 / 4-e^{-t}\right) x(g(t))\right)^{\Delta}\right]^{\Delta}} \\
& \quad+\Phi_{\gamma}\left(x^{\sigma}(t)\right)+\Phi_{\gamma}\left(x\left(\tau_{1}(t)\right)\right)-2^{-\gamma} \Phi_{\gamma}\left(x\left(\tau_{2}(t)\right)\right)=0,
\end{aligned}
$$

where $m_{1}=1-\left(1 / 4-e^{-t}\right) \geq 3 / 4$. We assume that $g(t)$ is rd-continuous on $[t, \infty)_{\mathbb{T}}$ which satisfies (B2). For $k_{1}, k_{2} \in \mathbb{N}$, and $k_{1} \geq k_{2}, \tau_{1}(t), \tau_{2}(t)$ are defined as follows:

$$
\tau_{1}(t)= \begin{cases}0 & \text { if } t \in \bigcup_{k=0}^{k_{1}}[k(a+b), k(a+b)+a], \\ t-k_{1}(a+b) & \text { if } t \in \bigcup_{k=k_{1}}^{\infty}[k(a+b), k(a+b)+a]\end{cases}
$$

and

$$
\tau_{2}(t)= \begin{cases}0 & \text { if } t \in \bigcup_{k=0}^{k_{2}}[k(a+b), k(a+b)+a], \\ t-k_{2}(a+b) & \text { if } t \in \bigcup_{k=k_{2}}^{\infty}[k(a+b), k(a+b)+a] .\end{cases}
$$

Then $\sigma(t)=t, t \in[k(a+b), k(a+b)+a), \sigma(t)=t+b, t=k(a+b)+a, k \in \mathbb{N}$. It is easy to check that conditions (B1)-(B6) and (2.2) hold,

$$
\left|\int^{\infty} \Phi_{\gamma}(3 / 4 \alpha)+\Phi_{\gamma}\left(3 / 4 \eta_{1} \alpha\right)-2^{-\gamma} \Phi_{\gamma}(\alpha) \Delta t\right|=\infty
$$

According to Corollary 3.1, any bounded solution of (4.3) is oscillatory.

Similar to Example 4.1, for any $\gamma>0$, it is easy to check that condition $\left(C_{1}\right)(\gamma>1)$ and (3.19)-(3.20) $(0<\gamma \leq 1)$ hold, respectively. Then we see that (4.3) is oscillatory.

In particular, (4.3) becomes the classical differential equations if $b=0$.

Example 4.3 Let $\Phi_{*}(u)=|u|^{*-1} u$ and time scale $\mathbb{T}=\mathbb{R}$. Consider the differential equation

$$
\begin{aligned}
& \left(x(t)+r_{0} x(g(t))\right)^{\prime \prime}+q_{0} \Phi_{2}(x(t))+q_{1} \Phi_{2}\left(x\left(\tau_{1}(t)\right)\right)-q_{2} \Phi_{2}\left(x\left(\tau_{2}(t)\right)\right) \\
& +s_{1} \Phi_{2}\left(x\left(\xi_{1}(t)\right)\right)-s_{2} \Phi_{2}\left(x\left(\xi_{2}(t)\right)\right)=0,
\end{aligned}
$$


where $r_{0}=1 / 3, m_{1}=1-r_{0}=2 / 3, g(t)=t-\ln t, \tau_{1}(t)=t-12, \tau_{2}(t)=t-1-\sin t / 4, \xi_{1}(t)=2 t$, $\xi_{2}(t)=t+3, q_{0}=10, q_{1}=8, q_{2}=7, s_{1}=36, s_{2}=5$. It is not difficult to check that conditions (B1)-(B6) hold. Since $s_{1} \Phi_{2}\left(m_{1}\right)-q_{2}-s_{2}=4>0$, it is easy to see that (2.3) and (3.17) hold. Similar to Example 4.1, we see that the function

$$
\begin{aligned}
h\left(t, x_{0}, x_{1}, x_{2}, x_{3}, x_{4}\right)= & q_{0} \Phi_{2}(x(t))+q_{1} \Phi_{2}\left(x\left(\tau_{1}(t)\right)\right)-q_{2} \Phi_{2}\left(x\left(\tau_{2}(t)\right)\right) \\
& +s_{1} \Phi_{2}\left(x\left(\xi_{1}(t)\right)\right)-s_{2} \Phi_{2}\left(x\left(\xi_{2}(t)\right)\right)
\end{aligned}
$$

satisfies condition $\left(C_{1}\right)$. Finally, we show that (3.12) holds. By Lemma 2.1(iii),

$$
\eta_{1}=\eta_{1}(t, T)=\frac{\tau_{1}(t)-\tau_{1}(T)}{t-T} \sim \frac{\tau_{1}(t)}{t}=\frac{t-\ln t}{t} \sim 1, \quad t \gg T
$$

so (3.12) holds. For any $\alpha \neq 0$,

$$
\begin{aligned}
\left|\int^{\infty} P(t, a) h\left(t, \alpha, \alpha \eta_{1}, 0, \alpha, 0\right) d t\right| & =\left|\int^{\infty}(t-a)\left[10 \Phi_{2}(\alpha)+8 \Phi_{2}(\alpha)+36 \Phi_{2}(\alpha)\right] d t\right| \\
& =\infty .
\end{aligned}
$$

By Theorem 3.5, (4.4) is oscillatory.

\section{Competing interests}

The authors declare that they have no competing interests.

\section{Authors' contributions}

The first author discovered the topic, offered the main ideas for the proof of the paper, and carried out writing this article. The first and the second authors discussed the paper together, and all the authors interchanged ideas about this paper. The second and the third authors gave some helpful suggestions for writing the paper and checked the proof of the paper. All authors read and approved the final manuscript.

\section{Author details}

${ }^{1}$ School of Mathematics and Computational Science, Sun Yat-Sen University, West Xingang Road 135, Guangzhou, 510275, P.R. China. ${ }^{2}$ Department of Mathematics, Texas A\&M University-Kingsville, 700 University Blvd., Kingsville, TX 78363-8202, USA. ${ }^{3}$ Department of Mathematics, King Abdulaziz University, P.O. Box 80203, Jeddah, 21589,

Saudi Arabia.

\section{Acknowledgements}

The authors would like to express their great gratitude to the anonymous valuable suggestions and comments, which helped the authors to improve the previous version of this article. This work was supported by the NNSF of P.R. China (Grant No. 11271379)

Received: 11 February 2014 Accepted: 22 April 2014 Published: 06 May 2014

\section{References}

1. Agarwal, RP, Grace, SR, O'Regan, D: Oscillation Theory for Second Order Dynamic Equations. Series in Mathematical Analysis and Applications, vol. 5. Taylor \& Francis, London (2003)

2. Bohner, M, Peterson, A: Dynamic Equations on Time Scales: An Introduction with Applications. Birkhäuser, Boston (2001)

3. Bohner, M, Peterson, A (eds.): Advances in Dynamic Equations on Time Scales. Birkhäuser, Boston (2003)

4. Saker, SH: Oscillation Theory of Dynamic Equations on Time Scales: Second and Third Orders. Lambert Academic Publishing, Saarbrücken (2010)

5. Agarwal, RP, Bohner, M, O'Regan, D, Peterson, A: Dynamic equations on time scales: a survey. J. Comput. Appl. Math. $141,1-26(2002)$

6. Agarwal, RP, Bohner, M, Řehák, P: Half-linear dynamic equations. In: Nonlinear Analysis and Applications: to V. Lakshmikantham on His 80th Birthday, vol. 1, pp. 1-57. Kluwer Academic, Dordrecht (2003)

7. Deng, XH, Wang, QR: Oscillation and nonoscillation for second-order nonlinear neutral functional dynamic equations on time scales. Electron. J. Differ. Equ. 2013, 234 (2013)

8. El-Morshedy, HA: New oscillation criteria for second order linear difference equations with positive and negative coefficients. Comput. Math. Appl. 58, 1988-1997 (2009) 
9. Grace, SR, Agarwal, RP, Bohner, M, O'Regan, D: Oscillation of second-order strongly superlinear and strongly sublinear dynamic equations. Commun. Nonlinear Sci. Numer. Simul. 14, 3463-3471 (2009)

10. Higgins, R: Some oscillation results for second order functional dynamic equations. Adv. Dyn. Syst. Appl. 5, 87-105 (2010)

11. Karpuz, B: Unbounded oscillation of higher-order nonlinear delay dynamic equations of neutral type with oscillating coefficients. Electron. J. Qual. Theory Differ. Equ. 2009, 34 (2009)

12. Manojlovic, J, Shoukaku, Y, Tanigawa, T, Yoshida, N: Oscillation criteria for second order differential equations with positive and negative coefficients. Appl. Math. Comput. 181, 853-863 (2006)

13. Özbekler, A, Zafer, A: Second order oscillation of mixed nonlinear dynamic equations with several positive and negative coefficients. Discrete Contin. Dyn. Syst., Dynamical systems, differential equations and applications. 8th AIMS Conference. Suppl. Vol. II, 1167-1175 (2011). ISBN:978-1-60133-008-6; ISBN:1-60133-008-1

14. Özbekler, A, Wong, JSW, Zafer, A: Forced oscillation of second order nonlinear differential equations with positive and negative coefficients. Appl. Math. Lett. 24, 1125-1130 (2011)

15. Tang, XH, Yu, JS, Peng, DH: Oscillation and nonoscillation of neutral difference equations with several positive and negative coefficients. Comput. Math. Appl. 39(7-8), 169-181 (2000)

16. Thandapani, E, Muthulakshmi, V, Graef, JR: Oscillation criteria for second order nonlinear neutral delay differential equations with positive and negative coefficients. Int. J. Pure Appl. Math. 70, 261-274 (2011)

17. Weng, A, Sun, J: Oscillation of second order delay differential equations. Appl. Math. Comput. 198, $930-935$ (2008)

18. Bai, Y, Liu, L: New oscillation criteria for second-order neutral delay differential equations with positive and negative coefficients. Abstr. Appl. Anal. 2010, Article ID 564068 (2010)

10.1186/1687-1847-2014-115

Cite this article as: Deng et al: Oscillation and nonoscillation for second order neutral dynamic equations with positive and negative coefficients on time scales. Advances in Difference Equations 2014, 2014:115

\section{Submit your manuscript to a SpringerOpen ${ }^{\circ}$ journal and benefit from:}

- Convenient online submission

- Rigorous peer review

- Immediate publication on acceptance

- Open access: articles freely available online

- High visibility within the field

- Retaining the copyright to your article 